\title{
Artificial sweeteners in pig feed: A worldwide survey and a case study in pig farms in Tianjin, China
}

Dandan Li,${ }^{\dagger}$ Yiming Yao, ${ }^{\dagger}$ Hongwen Sun,,${ }^{*}$ Yu Wang,,${ }^{\dagger}$ Jian Pu, ${ }^{\S}$ Raul Calderón, ${ }^{\#}$ Alfredo C. Alder, ${ }^{\dagger}$ Kurunthachalam Kannan*

${ }^{\dagger}$ MOE Key Laboratory of Pollution Processes and Environmental Criteria, College of Environmental Science and Engineering, Nankai University, Tianjin 300350, China

\#Centro de Investigación en Recursos Naturales y Sustentabilidad, Universidad Bernardo O’Higgins, Fabrica 1990, Segundo Piso, Santiago, Chile.

*Wadsworth Center, New York State Department of Health, and Department of Environmental Health Sciences, School of PublicHealth, State University of New York at Albany, Albany, New York 12201, United States

${ }^{\S}$ Faculty of Information Networking for Innovation and Design, Toyo University, Tokyo 115-0053, Japan; Institute for Future Initiatives, The University of Tokyo, Tokyo 113-8654, Japan

38 Tongyan Road, Jinnan District, Tianjin 300350, China

\section{$\underline{\text { Tel:86-22-23509241 }}$}

E-mail: sunhongwen@nankai.edu.cn

\section{Supporting Information}

List of Supporting Information

Text S1 Composting procedure in pig farms

Text S2 Instrumental analysis

Text S3 Recovery calculation

Figure S1. Technology flow chart of WWTP for YX pig farm.

Figure S2. Technology flow chart of WWTP for YLL pig farm.

Figure S3. Technology flow chart of WWTPs for other 14 pig farms studied.

Figure S4. Technology flow chart of pig manure composting at pig farms.

Figure S5. Correlations of the ASs concentrations between pig feed and manure samples.

Table S1. Structures and physicochemical properties of the seven artificial sweeteners used in this study. 
Table S2. The commercial sources of the target analytes and corresponding surrogates used to normalize each target analyte.

Table S3. Geographic information and culture scale of studied pig farms in Tianjin, China.

Table S4. Parameters for ASs mass loads calculation per capita of three kinds of pigs.

Table S5. Recoveries for ASs in investigated samples.

Table S6. Identification, quantification ions and MDLs for test ASs.

Table S7. Concentrations $(\mathrm{mg} / \mathrm{kg})$ of ASs in pig feeds samples and paired manure samples in Tianjin China.

Table S8. Concentrations ( $\mu \mathrm{g} / \mathrm{L})$ and removal efficiency of ASs in wastewater samples in on-farm WWTPs in Tianjin, China.

Table S9. ASs levels in wastewater samples in the present study and literature globally.

Table S10. Concentrations ( $\mathrm{mg} / \mathrm{kg}$ ) of ASs in compost samples from pig farms in Tianjin, China.

Table S11. Concentrations $(\mu \mathrm{g} / \mathrm{kg})$ of ASs in soil samples from pig farms in Tianjin, China.

Table S12. Pearson correlations of ASs in soil with pig farms of different scale.

Table S13. Sampling sites and characterization of soils in the literature.

Table S14. Annual mass loads of ASs ( $\mathrm{kg} / 10^{3}$ capita) in pig feeds samples and manure samples in Tianjin China.

Table S15. Concentrations ( $\mathrm{mg} / \mathrm{kg}$ ) of ASs in pig feeds samples from different countries. 


\section{Text S1 Composting procedure in pig farms}

Static aerobic composting technology was applied in the pig farms, mainly involving natural aeration combined with turning by forklift. The manure was mixed with corn straw at proper ratios to get a $\mathrm{C} / \mathrm{N}$ ratio of about $20-30$ to constitute a compost mixture. The compost mixture was initially piled into wedge shapes (approximately (2-10) $\mathrm{m}$ long $\times(1-2) \mathrm{m}$ width $\times(0.5-2) \mathrm{m}$ height $)$ for two weeks and then changed into conical shapes ((1-5) m diameter $\times(1-3) \mathrm{m}$ height $)$ for another three to five weeks (Figure S4).

\section{Text S2 Instrumental analysis}

Agilent HPLC 1260 system (Agilent Technologies, CA, USA) liquid chromatography-triple quadrupole mass spectrometry (LC-MS/MS) coupled with an Agilent 6460B triple quadrupole mass spectrometer (Agilent Technologies, CA, USA) equipped with an electrospray ionization (ESI) interface operated in negative ionization multiple-reaction monitoring mode was used for analysis of ASs. Nitrogen (with a purity of 99.9\%) was used as desolvation gas, with a manipulating temperature of $350{ }^{\circ} \mathrm{C}$. The flow rate was $10 \mathrm{~L} / \mathrm{min}$, and the nebulizing gas pressure was 50 psi. Separations were performed using an Athena C18-WP column $(4.6 \mathrm{~mm} \times$ $150 \mathrm{~mm}, 3 \mu \mathrm{m})(\mathrm{CNW}$ Technologies GmbH, Germany), which is compatible with a $100 \%$ aqueous mobile phase. The column was kept at $30{ }^{\circ} \mathrm{C}$. The mobile phase was composed of water and acetonitrile, both containing $5 \mathrm{mM}$ ammonium acetate and 1 mM TRIS. 


\section{Text S3 Recovery calculation}

The recovery for the seven ASs was evaluated as following: Blank group (A): real sample matrices; Pre-spiked group (B): real sample matrices spiked with native analytes before extraction; Post-spiked group (C): real sample matrices spiked with native analytes after extraction. Internal standards were spiked after extraction consistently for all the groups above to obtain the corrected value. The recovery was then calculated as follows:

$$
\text { Recovery }(\%)=\frac{C-A}{B-A} \times 100 \%
$$




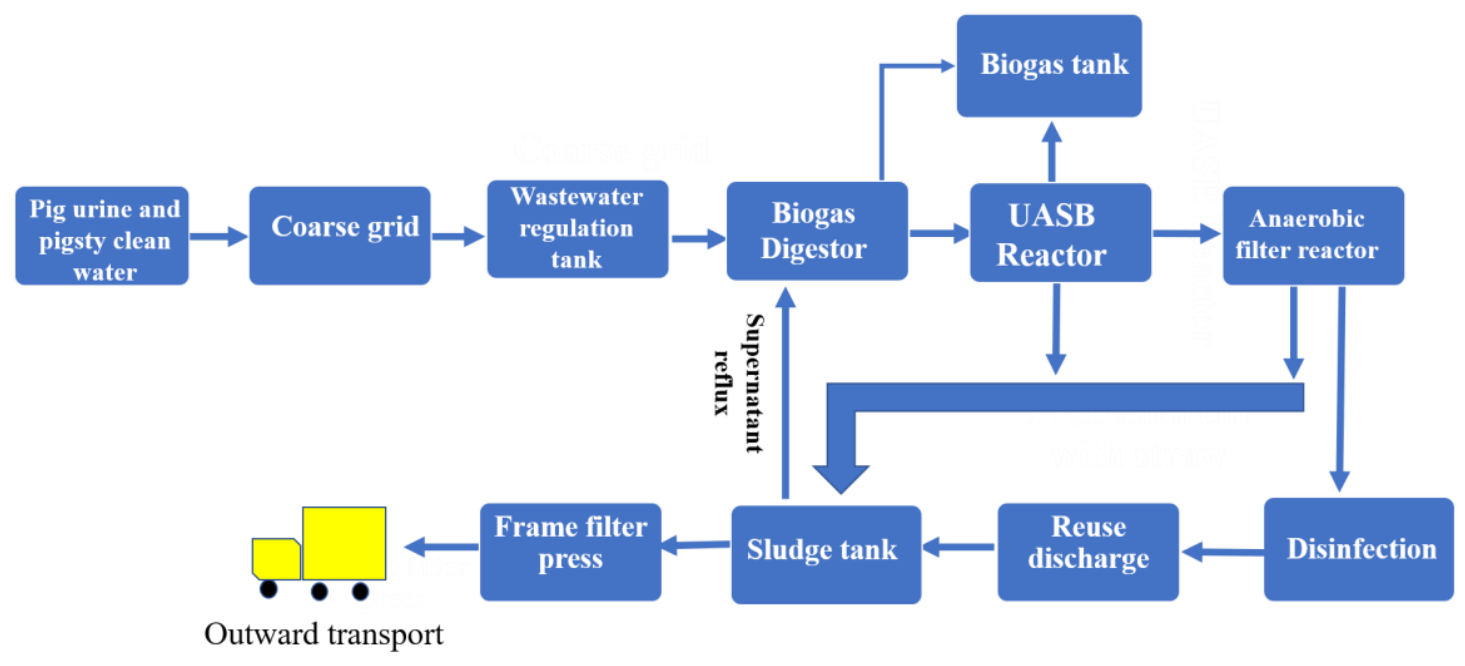

Figure S1. Technology flow chart of WWTP for YX pig farm.

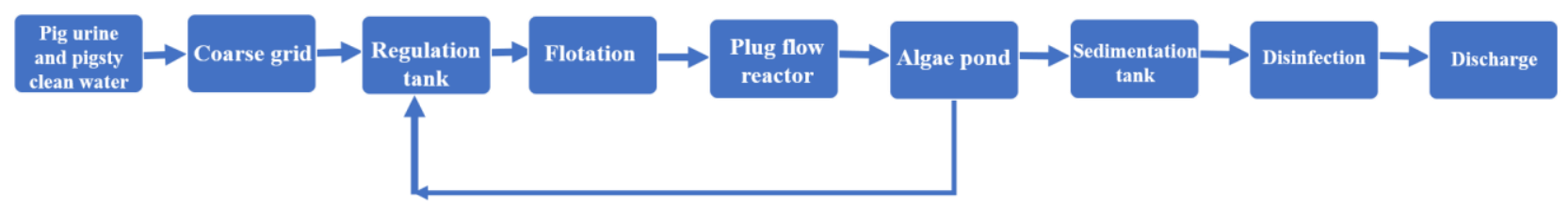

Figure S2. Technology flow chart of WWTP for YLL pig farm.

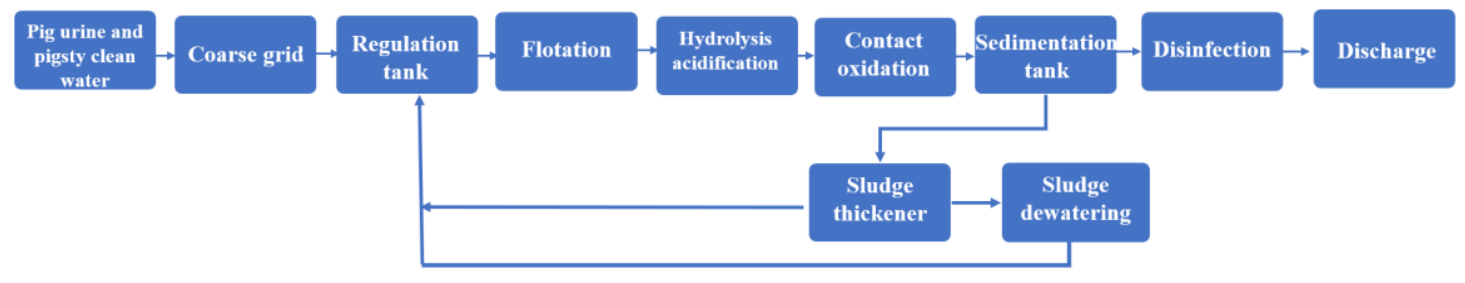

Figure S3. Technology flow chart of WWTPs for other 14 pig farms studied. 


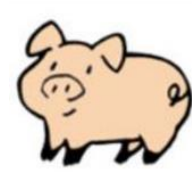

Pretreatment
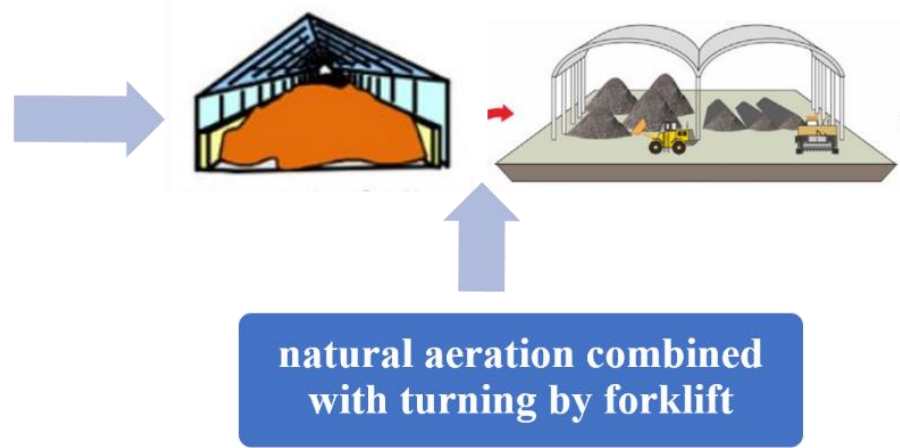

Pig manure

Figure S4. Technology flow chart of pig manure composting at pig farms.
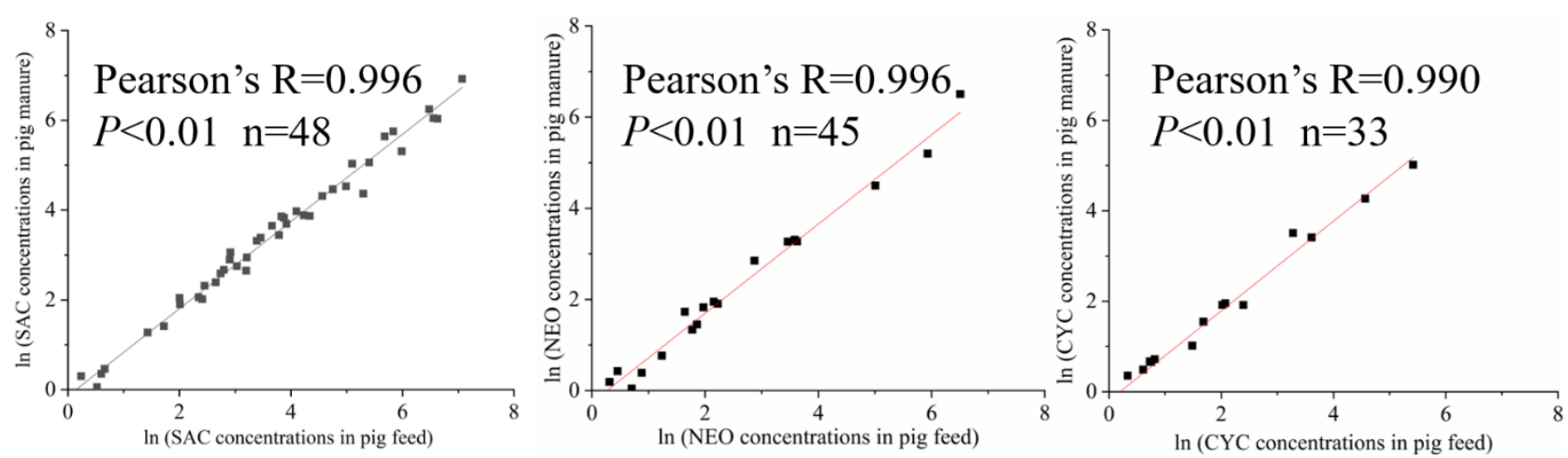

Figure S5. Correlations of the ASs concentrations between pig feed and manure samples. 
Table S1. Structures and physicochemical properties of the seven artificial sweeteners used in this studya.

\begin{tabular}{|c|c|c|c|c|c|c|c|}
\hline & SAC & CYC & $\mathrm{ACE}$ & SUC & ASP & $\mathrm{NEO}$ & NHDC \\
\hline \multicolumn{8}{|l|}{ Structure } \\
\hline Molecular weight $(\mathrm{g} / \mathrm{mol})$ & 183.2 & 179.2 & 163.2 & 397.6 & 294.3 & 378.5 & 612.6 \\
\hline Sugar equivalence & 300 & 30 & 200 & 600 & $160-220$ & $7000-13000$ & 1800 \\
\hline Solubility(g/L) & 4 & 1000 & 270 & 283 & 10 & 12.6 & 0.4 \\
\hline $\mathrm{p} K_{\mathrm{a}}$ & 2.2 & 1.9 & 2 & 11.8 & 3.19 and 7.87 & 3.01 and 8.02 & 9.7 \\
\hline $\log K_{\mathrm{ow}}$ & 0.91 & -1.61 & -1.33 & -1 & 0.07 & 2.39 & 0.75 \\
\hline Vapour pressure (mm Hg) & $1.0 \times 10^{-7}$ & $5.3 \times 10^{-7}$ & $9.0 \times 10^{-6}$ & $3.2 \times 10^{-14}$ & $2.8 \times 10^{-11}$ & $2.7 \times 10^{-11}$ & 1 \\
\hline
\end{tabular}

a: (TOXNET, 2017) 
Table S2.The commercial sources of the target analytes and corresponding surrogates used to normalize each target analyte.

\begin{tabular}{|c|c|c|c|}
\hline Chemicals & Abbreviation & Surrogates normalized by & Supplier \\
\hline saccharin & SAC & SAC-d4 & Sigma-Aldrich (St. Louis, MO, USA) \\
\hline cyclamate & $\mathrm{CYC}$ & ACE-d4 & Sigma-Aldrich (St. Louis, MO, USA) \\
\hline acesulfame & $\mathrm{ACE}$ & ACE-d4 & Sigma-Aldrich (St. Louis, MO, USA) \\
\hline neohesperidin dihydrochalcone & NHDC & SUC-d6 & Sigma-Aldrich (St. Louis, MO, USA) \\
\hline sucralose & SUC & SUC-d6 & Sigma-Aldrich (St. Louis, MO, USA) \\
\hline aspartame & ASP & ASP-d5 & Supelco (Bellefonte, PA, USA); \\
\hline neotame & NEO & NEO-d3 & USP Reference Standards (Rockville, MD, USA) \\
\hline Saccharin-d4 & SAC-d4 & & Toronto Research Chemicals (North York, ON, Canada) \\
\hline Acesulfame-d4 & ACE-d4 & & Toronto Research Chemicals (North York, ON, Canada) \\
\hline Sucralose-d6 & SUC-d6 & & Toronto Research Chemicals (North York, ON, Canada) \\
\hline Aspartame-d5 & ASP-d5 & & Toronto Research Chemicals (North York, ON, Canada) \\
\hline Neotame-d3 & NEO-d3 & & Toronto Research Chemicals (North York, ON, Canada) \\
\hline hydroxymethyl aminomethane & TRIS & & Sigma-Aldrich (St. Louis, MO, USA) \\
\hline
\end{tabular}


Table S3. Geographic information and culture scale, and sample types of the studied pig farms in Tianjin, China.

\begin{tabular}{|c|c|c|c|c|c|c|c|c|c|}
\hline \multirow[b]{2}{*}{ Name } & \multirow{2}{*}{$\begin{array}{c}\text { GPS } \\
\text { coordinate }\end{array}$} & \multicolumn{3}{|c|}{ Number (annual) } & \multicolumn{5}{|c|}{ Sampling } \\
\hline & & sow & piglet & hog & Feed & Manure & $\begin{array}{l}\text { Waste } \\
\text { water }\end{array}$ & Compost & Soil \\
\hline JHS & $\begin{array}{c}38^{\circ} 52^{\prime} 53^{\prime \prime N} \\
117^{\circ} 3^{\prime} 7^{\prime \prime} \mathrm{E}\end{array}$ & 200 & 3500 & 3000 & $\sqrt{ }$ & $\sqrt{ }$ & $\sqrt{ }$ & $\sqrt{ }$ & $\sqrt{ }$ \\
\hline $\mathrm{ZY}$ & $\begin{array}{c}38^{\circ} 42^{\prime} 56^{\prime \prime N} \\
117^{\circ} 8^{\prime} 12^{\prime \prime} \mathrm{E}\end{array}$ & 100 & 1800 & 1500 & $\sqrt{ }$ & $\sqrt{ }$ & $\sqrt{ }$ & $\sqrt{ }$ & $\sqrt{ }$ \\
\hline DMY & $\begin{array}{l}38^{\circ} 52^{\prime} 26^{\prime \prime} \mathrm{N} \\
116^{\circ} 53^{\prime} 20^{\prime \prime} \mathrm{E}\end{array}$ & 50 & 900 & 800 & $\sqrt{ }$ & $\sqrt{ }$ & $\sqrt{ }$ & $\sqrt{ }$ & $\sqrt{ }$ \\
\hline YXS & $\begin{array}{l}38^{\circ} 52^{\prime} 9^{\prime \prime} \mathrm{N} \\
116^{\circ} 56^{\prime} 39^{\prime \prime} \mathrm{E}\end{array}$ & 40 & 700 & 640 & $\sqrt{ }$ & $\sqrt{ }$ & $\sqrt{ }$ & $\sqrt{ }$ & $\sqrt{ }$ \\
\hline $\mathrm{XR}$ & $\begin{array}{l}38^{\circ} 51^{\prime} 35^{\prime \prime N} \\
116^{\circ} 533^{\prime} 57^{\prime \prime} \mathrm{E}\end{array}$ & 200 & 3500 & 3000 & $\sqrt{ }$ & $\sqrt{ }$ & $\sqrt{ }$ & $\sqrt{ }$ & $\sqrt{ }$ \\
\hline YF & $\begin{array}{c}38^{\circ} 47^{\prime} 43^{\prime \prime N} \\
117^{\circ} 12^{\prime} 4^{\prime \prime} \mathrm{E}\end{array}$ & 100 & 1800 & 1600 & $\sqrt{ }$ & $\sqrt{ }$ & $\sqrt{ }$ & $\sqrt{ }$ & $\sqrt{ }$ \\
\hline ZYY & $\begin{array}{l}38^{\circ} 49^{\prime} 41^{\prime \prime N} \\
116^{\circ} 58^{\prime} 38^{\prime \prime} \mathrm{E}\end{array}$ & 1600 & 28000 & 25000 & $\sqrt{ }$ & $\sqrt{ }$ & $\sqrt{ }$ & $\sqrt{ }$ & $\sqrt{ }$ \\
\hline $\mathrm{TZ}$ & $\begin{array}{l}38^{\circ} 43^{\prime} 53^{\prime \prime N} \\
117^{\circ} 14^{\prime} 10^{\prime \prime} \mathrm{E}\end{array}$ & 2400 & 43000 & 38000 & $\sqrt{ }$ & $\sqrt{ }$ & $\sqrt{ }$ & & \\
\hline $\mathrm{JF}$ & $\begin{array}{l}39^{\circ} 35^{\prime} 21^{\prime \prime N} \\
117^{\circ} 14^{\prime} 10^{\prime \prime}\end{array}$ & 3000 & 54000 & 48000 & $\sqrt{ }$ & $\sqrt{ }$ & $\sqrt{ }$ & $\sqrt{ }$ & $\sqrt{ }$ \\
\hline HT & $\begin{array}{l}39^{\circ} 35^{\prime} 20^{\prime \prime} \mathrm{N} \\
117^{\circ} 25^{\prime} 39^{\prime \prime} \mathrm{E}\end{array}$ & 5000 & 90000 & 80000 & $\sqrt{ }$ & $\sqrt{ }$ & $\sqrt{ }$ & & \\
\hline NK & $\begin{array}{l}39^{\circ} 41^{\prime} 30^{\prime \prime} \mathrm{N} \\
117^{\circ} 24^{\prime} 43^{\prime \prime} \mathrm{E}\end{array}$ & 1000 & 18000 & 16000 & $\sqrt{ }$ & $\sqrt{ }$ & $\sqrt{ }$ & & \\
\hline YX & $\begin{array}{l}39^{\circ} 25^{\prime} 20^{\prime \prime} \mathrm{N} \\
117^{\circ} 40^{\prime} 20^{\prime \prime} \mathrm{E}\end{array}$ & 2000 & 36000 & 32000 & $\sqrt{ }$ & $\sqrt{ }$ & $\sqrt{ }$ & $\sqrt{ }$ & $\sqrt{ }$ \\
\hline YLL & $\begin{array}{c}39^{\circ} 8^{\prime} 4^{\prime \prime N} \\
116^{\circ} 58^{\prime} 36^{\prime \prime}\end{array}$ & 700 & 12000 & 10000 & $\sqrt{ }$ & $\sqrt{ }$ & $\sqrt{ }$ & $\sqrt{ }$ & $\sqrt{ }$ \\
\hline JH & $\begin{array}{l}39^{\circ} 10^{\prime} 27^{\prime \prime N} \\
116^{\circ} 57^{\prime} 47^{\prime \prime} \mathrm{E}\end{array}$ & 500 & 9000 & 8000 & $\sqrt{ }$ & $\sqrt{ }$ & $\sqrt{ }$ & & \\
\hline $\mathrm{TY}$ & $\begin{array}{c}39^{\circ} 48^{\prime} 52^{\prime \prime N} \\
117^{\circ} 9^{\prime} 58^{\prime \prime} \mathrm{E}\end{array}$ & 550 & 9500 & 8800 & $\sqrt{ }$ & $\sqrt{ }$ & $\sqrt{ }$ & & \\
\hline HK & $\begin{array}{l}39^{\circ} 63^{\prime} 12^{\prime \prime} \mathrm{N} \\
117^{\circ} 30^{\prime} 24^{\prime \prime} \mathrm{P}\end{array}$ & 600 & 10000 & 9000 & $\sqrt{ }$ & $\sqrt{ }$ & $\sqrt{ }$ & $\sqrt{ }$ & $\sqrt{ }$ \\
\hline Total & & 18040 & 321700 & 285340 & & & & & \\
\hline
\end{tabular}


Table S4. Parameters for ASs mass loads calculation per capita of three kinds of pigs.

\begin{tabular}{cccc}
\hline & piglet & hog & sow \\
\hline Feed consumption rate $(\mathrm{kg} / \mathrm{d})$ & 1 & 3 & 2.5 \\
Annual feed consumption amount $(\mathrm{kg} / \mathrm{y})\left(A_{\mathrm{c}}\right)$ & 365 & 1095 & 912 \\
Manure emission rate $(\mathrm{kg} / \mathrm{d}$ w.w) & $1-2$ & 8 & 10 \\
Manure emission rate $(\mathrm{kg} / \mathrm{d} \mathrm{d} . \mathrm{w})$ & $0.1-0.3$ & $0.8-1.2$ & $1-1.5$ \\
Mean value $(\mathrm{kg} / \mathrm{d})$ & 0.2 & 1 & 1.25 \\
Annual manure emission amount $(\mathrm{kg} / \mathrm{y})\left(A_{\mathrm{e}}\right)$ & 73 & 365 & 456 \\
\hline
\end{tabular}

w.w: wet weight; d.w: dry weight

Table S5. Recoveries for ASs in investigated samples.

\begin{tabular}{cccccccc}
\hline Recovery $(\mathrm{RSD}) \%(\mathrm{n}=3)$ & SAC & CYC & NEO & ACE & SUC & ASP & NHDC \\
\hline Feed samples & $90.9(1.4)$ & $86.1(2.3)$ & $98.6(4.7)$ & $88.2(1.5)$ & $84.4(0.6)$ & $56.9(7.3)$ & $84.5(1.8)$ \\
Manure samples & $72.2(4.1)$ & $89.4(1.9)$ & $93.0(1.4)$ & $87.9(0.4)$ & $72.4(3.1)$ & $56.7(8.1)$ & $92.1(5.6)$ \\
Soil samples & $94.0(3.4)$ & $104.4(2.3)$ & $96.1(2.7)$ & $94.6(6.1)$ & $89.7(1.7)$ & $54.2(3.0)$ & $80.6(1.4)$ \\
Compost samples & $93.0(3)$ & $96.0(2)$ & $91.0(3)$ & $90.7(1.6)$ & $84.2(1.8)$ & $62.9(4.1)$ & $89.4(2.6)$ \\
Wastewater samples & $90.3(6.5)$ & $80.0(2.4)$ & $97.6(2.5)$ & $89.2(2.9)$ & $81.6(3.0)$ & $65.3(4.2)$ & $89.2(2.7)$ \\
\hline
\end{tabular}

Table S6. Identification, quantification transitions and MDLs for the test ASs.

\begin{tabular}{ccccccc}
\hline ASs & Precursor & Product & Linearity $^{\mathrm{a}}$ & $\mathrm{LOQ}^{\mathrm{b}}$ & \multicolumn{2}{c}{ MDL $(\mathrm{ng} / \mathrm{g})$} \\
\cline { 5 - 7 } & ion & ion & $\mathrm{R}^{2}$ & $(\mathrm{ng} / \mathrm{L})$ & $\begin{array}{c}\text { Feed/manure/compost } \\
(0.1 \mathrm{~g})\end{array}$ & $\begin{array}{c}\text { soil } \\
(5 \mathrm{~g})\end{array}$ \\
\hline SAC & 182 & $42.1 / 105.9$ & 0.997 & 116 & 9.78 & 0.20 \\
$\mathrm{CYC}$ & 178.1 & 80 & 0.999 & 72.5 & 0.36 & 0.007 \\
NEO & 377.1 & $344.8 / 199.9$ & 0.998 & 6.00 & 0.03 & 0.001 \\
ACE & 162 & $82 / 78$ & 0.999 & 39 & 2.45 & 0.05 \\
NHDC & 611.1 & $302.7 / 124.4$ & 0.999 & 620 & 3.10 & 0.06 \\
SUC & 395.1 & $35.1 / 37.1$ & 0.992 & 1380 & 6.9 & 0.14 \\
ASP & 293.2 & $200.2 / 261.1$ & 0.998 & 129 & 0.65 & 0.01 \\
\hline
\end{tabular}

a: Eight-point calibration (1-200 ug/L, in methanol); b S/N=10 (signal/noise) 
Table S7. Concentrations (mg/kg) of ASs in pig feeds samples and paired manure samples in Tianjin China.

(n: pig feed=48, pig manure $=48$ )

\begin{tabular}{|c|c|c|c|c|c|c|c|c|c|c|c|c|c|c|c|}
\hline & & \multicolumn{2}{|c|}{ SAC } & \multicolumn{2}{|c|}{ NEO } & \multicolumn{2}{|c|}{$\mathrm{CYC}$} & \multicolumn{2}{|c|}{ ACE } & \multicolumn{2}{|c|}{ NHDC } & \multicolumn{2}{|c|}{ ASP } & \multicolumn{2}{|c|}{ SUC } \\
\hline & & feed & manure & feed & manure & feed & manure & feed & manure & feed & manure & feed & manure & feed & manure \\
\hline \multirow[t]{3}{*}{ JHS } & Piglet & $67.6 \pm 1.2$ & $87.0 \pm 6.2$ & $0.48 \pm 0.01$ & $0.45 \pm 0.02$ & N.D. & N.D. & N.D. & N.D. & N.D. & N.D. & N.D. & N.D. & N.D. & N.D. \\
\hline & Hog & $36.6 \pm 1.1$ & $41.8 \pm 3.7$ & $0.0490 \pm 0.0001$ & $0.060 \pm 0.001$ & N.D. & N.D. & N.D. & N.D. & N.D. & N.D. & N.D. & N.D. & N.D. & N.D. \\
\hline & Sow & $1.48 \pm 0.21$ & $1.26 \pm 0.02$ & $0.0460 \pm 0.0001$ & $0.040 \pm 0.001$ & N.D. & N.D. & N.D. & N.D. & N.D. & N.D. & N.D. & N.D. & N.D. & N.D. \\
\hline \multirow[t]{3}{*}{$\mathrm{ZY}$} & Piglet & $96.6 \pm 6.5$ & $121 \pm 4$ & $59.2 \pm 1.4$ & $66.1 \pm 1.3$ & $49.8 \pm 1.5$ & $72.6 \pm 2.7$ & N.D. & N.D. & $1.70 \pm 0.02$ & $2.85 \pm 0.01$ & N.D. & N.D. & N.D. & N.D. \\
\hline & Hog & $18.4 \pm 0.9$ & $20.2 \pm 1.7$ & $3.89 \pm 0.02$ & $4.65 \pm 0.02$ & $0.98 \pm 0.01$ & $0.96 \pm 0.02$ & N.D. & N.D. & $1.65 \pm 0.01$ & $1.76 \pm 0.01$ & N.D. & N.D. & N.D. & N.D. \\
\hline & Sow & $2.75 \pm 0.14$ & $2.11 \pm 0.01$ & $0.180 \pm 0.001$ & $0.150 \pm 0.001$ & $0.44 \pm 0.01$ & $0.33 \pm 0.01$ & N.D. & N.D. & $1.22 \pm 0.01$ & $1.06 \pm 0.01$ & N.D. & N.D. & N.D. & N.D. \\
\hline \multirow[t]{3}{*}{ DMY } & Piglet & $206 \pm 11$ & $232 \pm 14$ & $3.49 \pm 0.02$ & $2.92 \pm 0.02$ & N.D. & N.D. & N.D. & N.D. & N.D. & N.D. & N.D. & N.D. & N.D. & N.D. \\
\hline & Hog & $95.7 \pm 5.9$ & $89 \pm 7$ & $0.380 \pm 0.001$ & $0.420 \pm 0.002$ & N.D. & N.D. & N.D. & N.D. & N.D. & N.D. & N.D. & N.D. & N.D. & N.D. \\
\hline & Sow & $16.5 \pm 1.1$ & $15.7 \pm 1.8$ & $0.500 \pm 0.001$ & $0.430 \pm 0.002$ & N.D. & N.D. & N.D. & N.D. & N.D. & N.D. & N.D. & N.D. & N.D. & N.D. \\
\hline \multirow[t]{3}{*}{ YXS } & Piglet & $276 \pm 11$ & $401 \pm 15$ & N.D. & N.D. & N.D. & N.D. & N.D. & N.D. & N.D. & N.D. & N.D. & N.D. & N.D. & N.D. \\
\hline & Hog & $24.2 \pm 1.3$ & $25.4 \pm 3.4$ & N.D. & N.D. & N.D. & N.D. & N.D. & N.D. & N.D. & N.D. & N.D. & N.D. & N.D. & N.D. \\
\hline & Sow & $10.1 \pm 0.3$ & $7.29 \pm 0.54$ & N.D. & N.D. & N.D. & N.D. & N.D. & N.D. & N.D. & N.D. & N.D. & N.D. & N.D. & N.D. \\
\hline \multirow[t]{3}{*}{$\mathrm{XR}$} & Piglet & $118 \pm 10$ & $151 \pm 12$ & $16.4 \pm 0.4$ & $17.1 \pm 1.9$ & $21.0 \pm 0.8$ & $22.0 \pm 1.7$ & N.D. & N.D. & N.D. & N.D. & N.D. & N.D. & N.D. & N.D. \\
\hline & hog & $19.1 \pm 1.1$ & $20.1 \pm 2.2$ & $11.4 \pm 0.2$ & $10.2 \pm 0.8$ & $2.79 \pm 0.12$ & $3.11 \pm 0.01$ & N.D. & N.D. & N.D. & N.D. & N.D. & N.D. & N.D. & N.D. \\
\hline & sow & $10.1 \pm 0.6$ & $7.13 \pm 0.45$ & $13.4 \pm 0.3$ & $7.40 \pm 0.43$ & $0.56 \pm 0.01$ & $0.50 \pm 0.001$ & N.D. & N.D. & N.D. & N.D. & N.D. & N.D. & N.D. & N.D. \\
\hline \multirow[t]{3}{*}{ YF } & piglet & $15.6 \pm 0.4$ & $16.4 \pm 1.2$ & $0.640 \pm 0.002$ & $0.85 \pm 0.01$ & N.D. & N.D. & N.D. & N.D. & N.D. & N.D. & N.D. & N.D. & N.D. & N.D. \\
\hline & hog & $4.49 \pm 0.02$ & $4.51 \pm 0.11$ & $0.120 \pm 0.001$ & $0.14 \pm 0.01$ & N.D. & N.D. & N.D. & N.D. & N.D. & N.D. & N.D. & N.D. & N.D. & N.D. \\
\hline & sow & $2.30 \pm 0.01$ & $1.62 \pm 0.02$ & $0.0500 \pm 0.0001$ & $0.030 \pm 0.001$ & N.D. & N.D. & N.D. & N.D. & N.D. & N.D. & N.D. & N.D. & N.D. & N.D. \\
\hline \multirow[t]{3}{*}{ YLL } & piglet & $83.7 \pm 3.4$ & $123 \pm 12$ & $24.5 \pm 1.3$ & $40.1 \pm 1.3$ & $51.3 \pm 0.3$ & $70.2 \pm 3.6$ & $4.26 \pm 0.01$ & $7.92 \pm 1.12$ & N.D. & N.D. & $0.81 \pm 0.04$ & N.D. & N.D. & N.D. \\
\hline & hog & $42.8 \pm 1.7$ & $39.4 \pm 1.5$ & $13.9 \pm 1.0$ & $12.00 \pm 0.03$ & $0.52 \pm 0.02$ & $0.65 \pm 0.01$ & $2.86 \pm 0.01$ & $2.13 \pm 0.01$ & N.D. & N.D. & $1.80 \pm 0.51$ & $0.11 \pm 0.02$ & N.D. & N.D. \\
\hline & sow & $38.8 \pm 2.2$ & $20.2 \pm 1.0$ & $9.36 \pm 0.43$ & $5.45 \pm 0.02$ & $12.6 \pm 0.1$ & $10.10 \pm 0.02$ & N.D. & N.D. & N.D. & N.D. & N.D. & N.D. & N.D. & N.D. \\
\hline
\end{tabular}


Table S7 Continued

\begin{tabular}{|c|c|c|c|c|c|c|c|c|c|c|c|c|c|c|c|}
\hline & & \multicolumn{2}{|c|}{ SAC } & \multicolumn{2}{|c|}{ NEO } & \multicolumn{2}{|c|}{$\mathrm{CYC}$} & \multicolumn{2}{|c|}{ ACE } & \multicolumn{2}{|c|}{ NHDC } & \multicolumn{2}{|c|}{ ASP } & \multicolumn{2}{|c|}{ SUC } \\
\hline & & feed & manure & feed & manure & feed & manure & feed & manure & feed & manure & feed & manure & feed & manure \\
\hline \multirow[t]{3}{*}{$\mathrm{JH}$} & piglet & $177 \pm 13$ & $230 \pm 4$ & $0.290 \pm 0.001$ & $0.31 \pm 0.02$ & $0.35 \pm 0.02$ & $0.28 \pm 0.01$ & N.D. & N.D. & N.D. & N.D. & N.D. & N.D. & N.D. & N.D. \\
\hline & hog & $67.9 \pm 4.5$ & $52.8 \pm 3.2$ & $0.260 \pm 0.001$ & $0.24 \pm 0.01$ & $0.12 \pm 0.01$ & $0.13 \pm 0.01$ & N.D. & N.D. & N.D. & N.D. & N.D. & N.D. & N.D. & N.D. \\
\hline & sow & $36.4 \pm 2.2$ & $28.9 \pm 2.1$ & $0.0800 \pm 0.0001$ & $0.060 \pm 0.001$ & $0.070 \pm 0.001$ & $0.03 \pm 0.01$ & N.D. & N.D. & N.D. & N.D. & N.D. & N.D. & N.D. & N.D. \\
\hline \multirow[t]{3}{*}{$\mathrm{TY}$} & piglet & $121 \pm 9$ & $142 \pm 9$ & $15.1 \pm 0.8$ & $20.6 \pm 1.2$ & $19.2 \pm 1.2$ & $19.9 \pm 0.1$ & N.D. & N.D. & N.D. & N.D. & N.D. & N.D. & N.D. & N.D. \\
\hline & hog & $21.2 \pm 1.2$ & $20.7 \pm 1.0$ & $13.4 \pm 0.2$ & $13.6 \pm 0.1$ & $3.18 \pm 0.01$ & $3.52 \pm 0.09$ & N.D. & N.D. & N.D. & N.D. & N.D. & N.D. & N.D. & N.D. \\
\hline & sow & $11.3 \pm 0.6$ & $7.47 \pm 0.41$ & $14.5 \pm 0.1$ & $11.3 \pm 0.1$ & $0.550 \pm 0.001$ & $0.50 \pm 0.01$ & N.D. & N.D. & N.D. & N.D. & N.D. & N.D. & N.D. & N.D. \\
\hline \multirow[t]{3}{*}{ HK } & piglet & $76.7 \pm 1.2$ & $132 \pm 16$ & $0.46 \pm 0.01$ & $0.640 \pm 0.001$ & $0.64 \pm 0.11$ & $1.06 \pm 0.01$ & N.D. & N.D. & N.D. & N.D. & N.D. & N.D. & N.D. & N.D. \\
\hline & hog & $18.1 \pm 0.2$ & $15.8 \pm 0.3$ & $0.12 \pm 0.01$ & $0.120 \pm 0.001$ & $0.37 \pm 0.09$ & $0.14 \pm 0.01$ & N.D. & N.D. & N.D. & N.D. & N.D. & N.D. & N.D. & N.D. \\
\hline & sow & $1.55 \pm 0.01$ & $1.06 \pm 0.01$ & $0.090 \pm 0.001$ & $0.0600 \pm 0.0001$ & $0.10 \pm 0.09$ & $0.090 \pm 0.001$ & N.D. & N.D. & N.D. & N.D. & N.D. & N.D. & N.D. & N.D. \\
\hline \multirow[t]{3}{*}{$\mathrm{TZ}$} & piglet & $86.1 \pm 2.9$ & $102 \pm 8$ & $0.53 \pm 0.02$ & $0.34 \pm 0.02$ & $0.49 \pm 0.02$ & $0.46 \pm 0.07$ & N.D. & N.D. & N.D. & N.D. & N.D. & N.D. & N.D. & N.D. \\
\hline & hog & $4.70 \pm 0.02$ & $5.50 \pm 0.53$ & $0.130 \pm 0.001$ & $0.150 \pm 0.001$ & $0.18 \pm 0.02$ & $0.20 \pm 0.04$ & N.D. & N.D. & N.D. & N.D. & N.D. & N.D. & N.D. & N.D. \\
\hline & sow & $8.44 \pm 0.03$ & $7.52 \pm 0.65$ & $0.080 \pm 0.0001$ & $0.050 \pm 0.001$ & $0.13 \pm 0.01$ & $0.11 \pm 0.04$ & N.D. & N.D. & N.D. & N.D. & N.D. & N.D. & N.D. & N.D. \\
\hline \multirow[t]{3}{*}{ ZYY } & piglet & $119 \pm 9$ & $78.0 \pm 0.3$ & $0.0600 \pm 0.0001$ & $0.110 \pm 0.001$ & $0.18 \pm 0.01$ & $0.24 \pm 0.01$ & N.D. & N.D. & N.D. & N.D. & N.D. & N.D. & N.D. & N.D. \\
\hline & hog & $43.4 \pm 2.2$ & $51.1 \pm 2.2$ & $0.0500 \pm 0.0001$ & $0.050 \pm 0.001$ & $0.14 \pm 0.01$ & $0.14 \pm 0.01$ & N.D. & N.D. & N.D. & N.D. & N.D. & N.D. & N.D. & N.D. \\
\hline & sow & $12.8 \pm 0.9$ & $13.3 \pm 0.1$ & $0.0500 \pm 0.0001$ & $0.020 \pm 0.001$ & $0.13 \pm 0.01$ & $0.14 \pm 0.01$ & N.D. & N.D. & N.D. & N.D. & N.D. & N.D. & N.D. & N.D. \\
\hline \multirow[t]{3}{*}{ HT } & piglet & $140 \pm 11$ & $129 \pm 3$ & $69.7 \pm 0.5$ & $56.0 \pm 1.2$ & $41.8 \pm 1.5$ & $46.6 \pm 2.6$ & N.D. & N.D. & N.D. & N.D. & N.D. & N.D. & N.D. & N.D. \\
\hline & hog & $15.8 \pm 2.5$ & $17.9 \pm 1.2$ & $31.0 \pm 0.1$ & $38.1 \pm 1.2$ & $1.23 \pm 0.16$ & $1.90 \pm 0.11$ & N.D. & N.D. & N.D. & N.D. & N.D. & N.D. & N.D. & N.D. \\
\hline & sow & $7.04 \pm 0.67$ & $5.91 \pm 0.11$ & $33.2 \pm 0.3$ & $18.0 \pm 0.3$ & $0.46 \pm 0.11$ & $0.39 \pm 0.02$ & N.D. & N.D. & N.D. & N.D. & N.D. & N.D. & N.D. & N.D. \\
\hline \multirow[t]{3}{*}{ NK } & piglet & $70.9 \pm 2.9$ & $74 \pm 4$ & $0.51 \pm 0.01$ & $0.53 \pm 0.01$ & N.D. & N.D. & N.D. & N.D. & N.D. & N.D. & N.D. & N.D. & N.D. & N.D. \\
\hline & hog & $37.8 \pm 1.6$ & $43.6 \pm 2.1$ & $0.070 \pm 0.001$ & $0.080 \pm 0.001$ & N.D. & N.D. & N.D. & N.D. & N.D. & N.D. & N.D. & N.D. & N.D. & N.D. \\
\hline & sow & $1.41 \pm 0.01$ & $1.35 \pm 0.01$ & $0.050 \pm 0.001$ & $0.050 \pm 0.001$ & N.D. & N.D. & N.D. & N.D. & N.D. & N.D. & N.D. & N.D. & N.D. & N.D. \\
\hline
\end{tabular}


Table S7 Continued

\begin{tabular}{|c|c|c|c|c|c|c|c|c|c|c|c|c|c|c|c|}
\hline & & \multicolumn{2}{|c|}{ SAC } & \multicolumn{2}{|c|}{ NEO } & \multicolumn{2}{|c|}{ CYC } & \multicolumn{2}{|c|}{ ACE } & \multicolumn{2}{|c|}{ NHDC } & \multicolumn{2}{|c|}{ ASP } & \multicolumn{2}{|c|}{ SUC } \\
\hline & & feed & manure & feed & manure & feed & manure & feed & manure & feed & manure & feed & manure & feed & manure \\
\hline \multirow[t]{3}{*}{$\mathrm{JF}$} & piglet & $123 \pm 10$ & $104 \pm 6$ & $11.1 \pm 0.1$ & $14.1 \pm 1.3$ & $29.6 \pm 1.5$ & $36.8 \pm 2.4$ & $1.06 \pm 0.17$ & $1.52 \pm 0.26$ & N.D. & N.D. & N.D. & N.D. & N.D. & N.D. \\
\hline & hog & $50.3 \pm 2.7$ & $48.9 \pm 3.1$ & $0.40 \pm 0.01$ & $0.53 \pm 0.02$ & $0.16 \pm 0.01$ & $0.18 \pm 0.02$ & N.D. & N.D. & N.D. & N.D. & N.D. & N.D. & N.D. & N.D. \\
\hline & sow & $2.74 \pm 0.12$ & $2.57 \pm 0.03$ & $0.75 \pm 0.02$ & $0.35 \pm 0.01$ & $0.10 \pm 0.01$ & $0.15 \pm 0.03$ & N.D. & N.D. & N.D. & N.D. & N.D. & N.D. & N.D. & N.D. \\
\hline \multirow[t]{3}{*}{ YX } & piglet & $326 \pm 19$ & $327 \pm 20$ & $0.17 \pm 0.01$ & $0.30 \pm 0.01$ & $0.14 \pm 0.01$ & $0.20 \pm 0.02$ & $0.16 \pm 0.01$ & $0.19 \pm 0.01$ & N.D. & N.D. & N.D. & N.D. & N.D. & N.D. \\
\hline & hog & $136 \pm 9$ & $144 \pm 7$ & $0.13 \pm 0.01$ & $0.12 \pm 0.01$ & $0.14 \pm 0.01$ & $0.12 \pm 0.01$ & $0.14 \pm 0.02$ & $0.17 \pm 0.01$ & N.D. & N.D. & N.D. & N.D. & N.D. & N.D. \\
\hline & sow & $21.6 \pm 1.2$ & $19.3 \pm 0.1$ & $0.12 \pm 0.01$ & $0.10 \pm 0.01$ & $0.13 \pm 0.01$ & $0.11 \pm 0.01$ & $0.13 \pm 0.01$ & $0.11 \pm 0.01$ & N.D. & N.D. & N.D. & N.D. & N.D. & N.D. \\
\hline
\end{tabular}

Data are expressed as mean \pm SD. 
Table S8. Concentrations $(\mu \mathrm{g} / \mathrm{L})$ and removal efficiency of ASs in wastewater samples from on-farm WWTPs in Tianjin, China.

(n: influent samples=16, effluent samples=16).

\begin{tabular}{|c|c|c|c|c|c|c|c|c|c|c|c|c|c|c|c|c|}
\hline & \multirow[b]{2}{*}{ NAME } & \multicolumn{2}{|c|}{ SAC } & \multirow[b]{2}{*}{$\begin{array}{c}\text { Removal } \\
\text { efficiency } \\
(100 \%) \\
\end{array}$} & \multicolumn{2}{|c|}{ NEO } & \multicolumn{3}{|c|}{$\mathrm{CYC}$} & \multirow[b]{2}{*}{$\begin{array}{c}\text { Removal } \\
\text { efficiency } \\
(100 \%)\end{array}$} & \multicolumn{2}{|c|}{$\overline{\mathrm{ACE}}$} & \multicolumn{4}{|c|}{ NHDC } \\
\hline & & influent & effluent & & influent & effluent & $\begin{array}{c}\text { Removal } \\
\text { efficiency } \\
(100 \%)\end{array}$ & influent & effluent & & influent & effluent & $\begin{array}{c}\text { Removal } \\
\text { efficiency } \\
(100 \%)\end{array}$ & influent & effluent & $\begin{array}{c}\text { Removal } \\
\text { efficiency } \\
(100 \%) \\
\end{array}$ \\
\hline \multirow{6}{*}{ Small-scale } & JHS & $198 \pm 11$ & $12.1 \pm 1.3$ & 93.9 & $0.59 \pm 0.01$ & $0.17 \pm 0.01$ & 70.6 & N.D. & N.D. & 1 & N.D. & N.D. & $T$ & N.D. & N.D. & $T$ \\
\hline & $\mathrm{ZY}$ & $71.8 \pm 7.9$ & $6.24 \pm 0.97$ & 91.2 & $0.50 \pm 0.01$ & $0.12 \pm 0.01$ & 68.2 & $1.44 \pm 0.02$ & $0.19 \pm 0.01$ & 86.8 & N.D. & N.D. & 1 & $4.45 \pm 0.02$ & $1.25 \pm 0.03$ & 71.9 \\
\hline & DMY & $1110 \pm 22$ & $65.8 \pm 3.5$ & 94.1 & $0.13 \pm 0.01$ & $0.020 \pm 0.001$ & 84.8 & N.D. & N.D. & 1 & N.D. & N.D. & 1 & N.D. & N.D. & 1 \\
\hline & YXS & $858 \pm 17$ & $57.4 \pm 2.2$ & 93.3 & N.D. & N.D. & 1 & N.D. & N.D. & 1 & N.D. & N.D. & 1 & N.D. & N.D. & 1 \\
\hline & XR & $1440 \pm 33$ & $66.8 \pm 5.2$ & 95.4 & $0.15 \pm 0.01$ & $0.020 \pm 0.001$ & 87.7 & $7.40 \pm 1.21$ & $0.67 \pm 0.03$ & 91.0 & N.D. & N.D. & 1 & N.D. & N.D. & 1 \\
\hline & YF & $111 \pm 9$ & $10.2 \pm 1.2$ & 90.8 & $0.88 \pm 0.02$ & $0.26 \pm 0.01$ & 70.4 & N.D. & N.D. & 1 & N.D. & N.D. & 1 & N.D. & N.D. & 1 \\
\hline \multirow{4}{*}{ Medium-scale } & YLL & $1010 \pm 23$ & $11.7 \pm 1.0$ & 98.8 & $1.21 \pm 0.25$ & $0.12 \pm 0.01$ & 90.2 & $59.5 \pm 1.3$ & $0.56 \pm 0.01$ & 99.1 & $0.70 \pm 0.04$ & $0.50 \pm 0.01$ & 28.9 & N.D. & N.D. & 1 \\
\hline & $\mathrm{JH}$ & $961 \pm 18$ & $31.4 \pm 1.2$ & 96.7 & $0.85 \pm 0.11$ & $0.20 \pm 0.01$ & 77.1 & $10.3 \pm 0.5$ & $2.23 \pm 0.21$ & 78.3 & N.D. & N.D. & 1 & N.D. & N.D. & 1 \\
\hline & TY & $103 \pm 4$ & $5.24 \pm 1.54$ & 94.9 & $0.48 \pm 0.01$ & $0.15 \pm 0.01$ & 69.2 & $1.19 \pm 0.11$ & $0.24 \pm 0.01$ & 79.7 & N.D. & N.D. & 1 & N.D. & N.D. & 1 \\
\hline & $\mathrm{HK}$ & $1440 \pm 26$ & $10.2 \pm 0.6$ & 99.3 & $0.080 \pm 0.001$ & $0.020 \pm 0.001$ & 81.8 & $0.75 \pm 0.02$ & $0.060 \pm 0.001$ & 92.6 & N.D. & N.D. & 1 & N.D. & N.D. & 1 \\
\hline \multirow{6}{*}{ Large-scale } & $\mathrm{TZ}$ & $433 \pm 15$ & $40 \pm 2$ & 90.8 & $0.97 \pm 0.02$ & $0.21 \pm 0.05$ & 78.7 & $37.2 \pm 1.4$ & $4.65 \pm 0.52$ & 87.5 & N.D. & N.D. & 1 & N.D. & N.D. & 1 \\
\hline & ZYY & $1180 \pm 22$ & $103 \pm 6$ & 91.3 & $1.06 \pm 0.01$ & $0.34 \pm 0.03$ & 76.4 & $21.6 \pm 1.1$ & $2.16 \pm 0.12$ & 90.0 & N.D. & N.D. & 1 & N.D. & N.D. & 1 \\
\hline & HT & $1080 \pm 10$ & $1.82 \pm 0.12$ & 99.8 & $0.23 \pm 0.01$ & $0.03 \pm 0.001$ & 89.2 & $18.9 \pm 1.5$ & $0.50 \pm 0.01$ & 97.4 & N.D. & N.D. & 1 & N.D. & N.D. & 1 \\
\hline & NK & $43.2 \pm 3.8$ & $3.27 \pm 0.11$ & 92.4 & $0.46 \pm 0.02$ & $0.11 \pm 0.01$ & 75.6 & N.D. & N.D. & 1 & N.D. & N.D. & 1 & N.D. & N.D. & 1 \\
\hline & JF & $468 \pm 9$ & $36.1 \pm 2.3$ & 92.3 & $0.44 \pm 0.02$ & $0.12 \pm 0.01$ & 72.1 & $12.5 \pm 0.3$ & $1.05 \pm 0.01$ & 91.6 & $5.94 \pm 0.75$ & $1.25 \pm 0.37$ & 79.0 & N.D. & N.D. & 1 \\
\hline & $\mathrm{YX}$ & $150 \pm 7$ & $3.63 \pm 0.09$ & 97.6 & $0.69 \pm 0.04$ & $0.11 \pm 0.01$ & 84.7 & $7.71 \pm 0.03$ & $0.25 \pm 0.01$ & 96.7 & $8.68 \pm 0.01$ & $0.53 \pm 0.02$ & 93.9 & N.D. & N.D. & 1 \\
\hline
\end{tabular}

Data are expressed as mean $\pm \mathrm{SD}$ 
Table S9. ASs levels in wastewater samples in present study and literature globally.

\begin{tabular}{cccccc}
\hline $\begin{array}{c}\text { Target } \\
\text { Analytes }\end{array}$ & $\begin{array}{c}\text { Concentration } \\
\text { Influent }(\boldsymbol{\mu g} / \mathbf{L})\end{array}$ & $\begin{array}{c}\text { Concentration } \\
\text { Effluent }(\boldsymbol{\mu g} / \mathbf{L})\end{array}$ & Region & Country & Reference \\
\hline SAC & Range: $7.2-9.1$ & Mean:0.27 & Municipal WWTP & China & (Gan et al., 2013) \\
SAC & Median: 8.23 & Median: 0.202 & Municipal WWTP & China & (Yang et al., 2017) \\
SAC & Mean: 16.4 & Mean:0.42 & Municipal WWTP & USA & (Subedi et al., 2014) \\
SAC & Mean:50 & Mean:2.8 & Municipal WWTP & Germany & (Scheurer et al., 2009) \\
CYC & Median:0.0875 & 1 & Municipal WWTP & Singapore & (Tran et al., 2014) \\
SAC & Median:663 & Median:11.9 & On-farm WWTP & China & Present study \\
CYC & Mean: 11.2 & Mean: 0.80 & On-farm WWTP & China & Present study \\
\hline
\end{tabular}

Table S10. Concentrations (mg/kg) of ASs in compost samples from pig farms in Tianjin, China.(n: compost samples=11)

\begin{tabular}{|c|c|c|c|c|c|c|c|c|c|c|c|c|}
\hline \multirow{2}{*}{ Compound } & \multicolumn{11}{|c|}{ Sites } & \multirow{2}{*}{$\begin{array}{c}\text { Detection } \\
\text { frequency } \\
(\%)\end{array}$} \\
\hline & $\mathrm{JF}$ & YLL & JHS & $\mathrm{ZY}$ & ZYY & YF & YX & DMY & YXS & XR & $\mathrm{HK}$ & \\
\hline $\mathrm{SAC}$ & $59.6 \pm 3.2$ & $50.3 \pm 2.9$ & $30.7 \pm 1.4$ & $25.3 \pm 1.3$ & $3.44 \pm 0.22$ & $4.02 \pm 0.28$ & $94.2 \pm 3.9$ & $87.6 \pm 3.0$ & $23.1 \pm 1.2$ & $32.2 \pm 2.3$ & $15.3 \pm 1.2$ & 100 \\
\hline NEO & $0.42 \pm 0.01$ & $10.3 \pm 0.9$ & $0.16 \pm 0.01$ & $4.25 \pm 0.43$ & $0.04 \pm 0.001$ & $0.12 \pm 0.01$ & $0.10 \pm 0.01$ & $3.01 \pm 0.25$ & N.D. & $8.01 \pm 0.27$ & $0.10 \pm 0.01$ & 91 \\
\hline CYC & $0.27 \pm 0.01$ & $9.14 \pm 0.65$ & N.D. & $0.88 \pm 0.01$ & $0.12 \pm 0.01$ & N.D. & $0.13 \pm 0.01$ & N.D. & N.D. & $1.44 \pm 0.02$ & $0.22 \pm 0.01$ & 64 \\
\hline $\mathrm{ACE}$ & $1.15 \pm 0.04$ & $1.84 \pm 0.03$ & N.D. & N.D. & N.D. & N.D. & $0.15 \pm 0.01$ & N.D. & N.D. & N.D. & N.D. & 27 \\
\hline
\end{tabular}

Data are expressed as mean \pm SD

Table S11. Concentrations ( $\mu \mathrm{g} / \mathrm{kg}$ ) of ASs in soil samples from pig farms in Tianjin, China. (n: soil samples=11)

\begin{tabular}{|c|c|c|c|c|c|c|c|c|c|c|c|c|}
\hline \multirow{2}{*}{ Compound } & & \multicolumn{10}{|c|}{ Sites } & \multirow{2}{*}{$\begin{array}{c}\text { Detection } \\
\text { frequency } \\
(\%)\end{array}$} \\
\hline & $\mathrm{JF}$ & YLL & JHS & $\mathrm{ZY}$ & ZYY & YF & YX & DMY & YXS & XR & $\mathrm{HK}$ & \\
\hline SAC & $8.23 \pm 1.23$ & $17.2 \pm 0.7$ & $61.6 \pm 3.2$ & $60.8 \pm 2.7$ & $14.9 \pm 1.3$ & $20.8 \pm 1.3$ & $33.7 \pm 2.3$ & $127 \pm 10$ & $91.2 \pm 4.1$ & $108 \pm 10$ & $121 \pm 10$ & 100 \\
\hline CYC & $1.60 \pm 0.14$ & $2.16 \pm 0.11$ & N.D. & $8.32 \pm 2.71$ & $2.73 \pm 0.06$ & N.D. & $3.33 \pm 0.25$ & N.D. & N.D. & $3.57 \pm 0.05$ & $2.17 \pm 0.02$ & 64 \\
\hline $\mathrm{ACE}$ & $1.02 \pm 0.02$ & $3.12 \pm 0.09$ & N.D. & N.D. & N.D. & N.D. & $1.62 \pm 0.51$ & N.D. & N.D. & N.D. & N.D. & 27 \\
\hline
\end{tabular}


Table S12. Pearson correlations of ASs in soil with pig farm of different scales.

\begin{tabular}{ccccccc}
\hline & Total pigs & sow & piglet & hog & SAC & NEO \\
\hline SAC & -0.6 & -0.60 & -0.60 & -0.59 & & \\
NEO & 0.05 & 0.05 & 0.05 & 0.05 & -0.55 & \\
CYC & -0.52 & -0.53 & -0.52 & -0.52 & 0.18 & 0.34
\end{tabular}

*Correlation is significant at the 0.01 level (2-tailed)

**Correlation is significant at the 0.05 level (2-tailed)

Table S13. Sampling sites and characterization of soils in the literature ${ }^{15}$.

\begin{tabular}{cccc}
\hline Soil site & texture & Organic matter content $(\%)$ & $\mathrm{pH}$ \\
\hline Arable land & loam & 1.9 & 7.2 \\
Arable land & Clay loam & 7.3 & 7.1 \\
Arable land & loam & 1.2 & 7.5 \\
Fertilized meadow & loam & 3.0 & 6.8 \\
Fertilized meadow & Sandy loam & 4.2 & 5.5 \\
Alpine pasture & clay & 18 & 4.0 \\
\hline
\end{tabular}


Table S14. Annual mass loads of ASs ( $\mathrm{kg} / 10^{3}$ capita) in pig feed and manure samples in Tianjin, China.

\begin{tabular}{|c|c|c|c|c|c|c|c|}
\hline & & \multicolumn{2}{|c|}{ SAC } & \multicolumn{2}{|c|}{$\mathrm{NEO}$} & \multicolumn{2}{|c|}{$\overline{\mathrm{CYC}}$} \\
\hline & & feed & manure & feed & manure & feed & manure \\
\hline \multirow[t]{3}{*}{ JHS } & Piglet & 24.7 & 6.35 & 0.18 & 0.03 & 0 & 0 \\
\hline & Hog & 40.1 & 15.3 & 0.05 & 0.02 & 0 & 0 \\
\hline & Sow & 1.35 & 0.57 & 0.04 & 0.02 & 0 & 0 \\
\hline \multirow[t]{3}{*}{$\mathrm{ZY}$} & Piglet & 35.3 & 8.83 & 21.6 & 4.83 & 18.2 & 5.30 \\
\hline & Hog & 20.1 & 7.37 & 4.26 & 1.70 & 1.70 & 0.35 \\
\hline & Sow & 2.51 & 0.96 & 0.16 & 0.07 & 0.40 & 0.15 \\
\hline \multirow[t]{3}{*}{ DMY } & Piglet & 75.2 & 16.9 & 1.27 & 0.21 & 0 & 0 \\
\hline & Hog & 105 & 32.5 & 0.42 & 0.15 & 0 & 0 \\
\hline & Sow & 15.0 & 7.16 & 0.45 & 0.20 & 0 & 0 \\
\hline \multirow[t]{3}{*}{ YXS } & Piglet & 101 & 29.3 & 0 & 0 & 0 & 0 \\
\hline & Hog & 26.5 & 9.27 & 0 & 0 & 0 & 0 \\
\hline & Sow & 9.21 & 3.32 & 0 & 0 & 0 & 0 \\
\hline \multirow[t]{3}{*}{ XR } & Piglet & 43.1 & 11.0 & 5.99 & 1.25 & 7.67 & 1.61 \\
\hline & hog & 20.9 & 7.34 & 12.5 & 3.72 & 3.06 & 1.14 \\
\hline & sow & 9.21 & 3.25 & 12.2 & 3.37 & 0.51 & 0.23 \\
\hline \multirow[t]{3}{*}{ YF } & piglet & 5.69 & 1.20 & 0.23 & 0.06 & 0 & 0 \\
\hline & hog & 4.92 & 1.65 & 0.13 & 0.05 & 0 & 0 \\
\hline & sow & 2.10 & 0.74 & 0.04 & 0.02 & 0 & 0 \\
\hline \multirow[t]{3}{*}{ YLL } & piglet & 30.6 & 8.98 & 8.94 & 2.93 & 18.7 & 5.12 \\
\hline & hog & 46.9 & 14.4 & 15.2 & 4.38 & 0.57 & 0.24 \\
\hline & sow & 35.4 & 9.21 & 8.54 & 2.49 & 11.5 & 4.61 \\
\hline \multirow[t]{3}{*}{$\mathrm{JH}$} & piglet & 64.6 & 16.8 & 0.11 & 0.02 & 0.13 & 0.02 \\
\hline & hog & 74.4 & 19.3 & 0.29 & 0.09 & 0.13 & 0.05 \\
\hline & sow & 33.2 & 13.2 & 0.07 & 0.03 & 0.07 & 0.02 \\
\hline \multirow[t]{3}{*}{$\mathrm{TY}$} & piglet & 44.2 & 10.4 & 5.51 & 1.50 & 7.0 & 1.45 \\
\hline & hog & 23.2 & 7.56 & 14.7 & 4.96 & 3.48 & 1.28 \\
\hline & sow & 10.3 & 3.41 & 13.2 & 5.15 & 0.50 & 0.23 \\
\hline \multirow[t]{3}{*}{$\mathrm{HK}$} & piglet & 28.0 & 9.64 & 0.17 & 0.05 & 0.23 & 0.08 \\
\hline & hog & 19.8 & 5.77 & 0.41 & 0.04 & 0.41 & 0.05 \\
\hline & sow & 1.41 & 0.48 & 0.08 & 0.03 & 0.09 & 0.04 \\
\hline \multirow[t]{3}{*}{$\mathrm{TZ}$} & piglet & 31.4 & 7.45 & 0.19 & 0.02 & 0.18 & 0.03 \\
\hline & hog & 5.15 & 2.01 & 0.15 & 0.05 & 0.20 & 0.07 \\
\hline & sow & 7.70 & 3.43 & 0.07 & 0.02 & 0.11 & 0.05 \\
\hline \multirow[t]{3}{*}{ ZYY } & piglet & 43.4 & 5.69 & 0.02 & 0.01 & 0.07 & 0.02 \\
\hline & hog & 47.5 & 18.7 & 0.05 & 0.02 & 0.16 & 0.05 \\
\hline & sow & 11.7 & 6.06 & 0.04 & 0.01 & 0.12 & 0.06 \\
\hline \multirow[t]{3}{*}{ HT } & piglet & 51.1 & 9.42 & 25.4 & 4.09 & 15.3 & 3.40 \\
\hline & hog & 17.3 & 6.53 & 33.9 & 13.9 & 1.35 & 0.69 \\
\hline & sow & 6.42 & 2.69 & 30.3 & 8.21 & 0.42 & 0.18 \\
\hline \multirow[t]{3}{*}{ NK } & piglet & 25.9 & 5.40 & 0.19 & 0.04 & 0 & 0 \\
\hline & hog & 41.4 & 15.9 & 0.07 & 0.03 & 0 & 0 \\
\hline & sow & 1.29 & 0.62 & 0.05 & 0.02 & 0 & 0 \\
\hline \multirow[t]{3}{*}{$\mathrm{JF}$} & piglet & 44.9 & 7.60 & 4.05 & 1.03 & 10.8 & 2.69 \\
\hline & hog & 55.1 & 17.8 & 0.44 & 0.19 & 0.18 & 0.07 \\
\hline & sow & 2.50 & 1.17 & 0.68 & 0.16 & 0.09 & 0.07 \\
\hline \multirow{3}{*}{ YX } & piglet & 119 & 23.9 & 0.06 & 0.02 & 0.05 & 0.01 \\
\hline & hog & 149 & 52.6 & 0.15 & 0.05 & 0.15 & 0.05 \\
\hline & sow & 19.7 & 8.80 & 0.11 & 0.05 & 0.12 & 0.05 \\
\hline
\end{tabular}


Table S15. Concentrations $(\mathrm{mg} / \mathrm{kg})$ of ASs in pig feed samples from different countries.

(n: USA=15, Switzerland=9, Chile=9, Japan=30)

\begin{tabular}{|c|c|c|c|c|c|c|c|c|c|}
\hline & No. & & SAC & NEO & $\mathrm{CYC}$ & $\mathrm{ACE}$ & NHDC & SUC & ASP \\
\hline \multirow{15}{*}{ USA } & 1 & hog & $2.29 \pm 0.24$ & N.D. & N.D. & N.D. & N.D. & N.D. & N.D. \\
\hline & 2 & piglet & $0.43 \pm 0.02$ & N.D. & N.D. & N.D. & N.D. & N.D. & N.D. \\
\hline & 3 & hog & $0.69 \pm 0.11$ & N.D. & N.D. & N.D. & N.D. & N.D. & N.D. \\
\hline & 4 & hog & $0.050 \pm 0.001$ & N.D. & N.D. & N.D. & N.D. & N.D. & N.D. \\
\hline & 5 & hog & $0.010 \pm 0.001$ & N.D. & N.D. & N.D. & N.D. & N.D. & N.D. \\
\hline & 6 & sow & $0.51 \pm 0.01$ & N.D. & N.D. & N.D. & N.D. & N.D. & N.D. \\
\hline & 7 & hog & $0.050 \pm 0.001$ & N.D. & N.D. & N.D. & N.D. & N.D. & N.D. \\
\hline & 8 & piglet & $0.30 \pm 0.01$ & N.D. & N.D. & N.D. & N.D. & N.D. & N.D. \\
\hline & 9 & piglet & $0.11 \pm 0.01$ & N.D. & N.D. & N.D. & N.D. & N.D. & N.D. \\
\hline & 10 & piglet & $0.85 \pm 0.02$ & N.D. & N.D. & N.D. & N.D. & N.D. & N.D. \\
\hline & 11 & sow & $0.040 \pm 0.001$ & N.D. & N.D. & N.D. & N.D. & N.D. & N.D. \\
\hline & 12 & piglet & $1.52 \pm 0.12$ & N.D. & N.D. & N.D. & N.D. & N.D. & N.D. \\
\hline & 13 & hog & $0.29 \pm 0.01$ & N.D. & N.D. & N.D. & N.D. & N.D. & N.D. \\
\hline & 14 & hog & $0.17 \pm 0.01$ & N.D. & N.D. & N.D. & N.D. & N.D. & N.D. \\
\hline & 15 & hog & $0.28 \pm 0.02$ & N.D. & N.D. & N.D. & N.D. & N.D. & N.D. \\
\hline \multirow{9}{*}{ Switzerland } & 1 & piglet & $83.9 \pm 1.3$ & N.D. & N.D. & N.D. & 1.32 & N.D. & N.D. \\
\hline & 2 & piglet & $71.6 \pm 0.7$ & N.D. & N.D. & N.D. & 0.70 & N.D. & N.D. \\
\hline & 3 & piglet & $95.2 \pm 1.0$ & N.D. & N.D. & N.D. & 1.01 & N.D. & N.D. \\
\hline & 4 & piglet & $89.7 \pm 0.1$ & N.D. & N.D. & N.D. & 0.09 & N.D. & N.D. \\
\hline & 5 & sow & $6.77 \pm 0.02$ & N.D. & N.D. & N.D. & 0.02 & N.D. & N.D. \\
\hline & 6 & hog & $0.710 \pm 0.002$ & N.D. & N.D. & N.D. & N.D. & N.D. & N.D. \\
\hline & 7 & piglet & $1.020 \pm 0.002$ & N.D. & N.D. & N.D. & N.D. & N.D. & N.D. \\
\hline & 8 & hog & $0.070 \pm 0.002$ & N.D. & N.D. & N.D. & N.D. & N.D. & N.D. \\
\hline & 9 & hog & $0.770 \pm 0.002$ & N.D. & N.D. & N.D. & N.D. & N.D. & N.D. \\
\hline \multirow{9}{*}{ Chile } & 1 & & $19.3 \pm 1.7$ & N.D. & N.D. & N.D. & N.D. & N.D. & N.D. \\
\hline & 2 & & $3.53 \pm 0.41$ & N.D. & N.D. & N.D. & N.D. & N.D. & N.D. \\
\hline & 3 & & $45.4 \pm 1.1$ & N.D. & N.D. & N.D. & N.D. & N.D. & N.D. \\
\hline & 4 & & $1.54 \pm 0.13$ & N.D. & N.D. & N.D. & N.D. & N.D. & N.D. \\
\hline & 5 & & $0.18 \pm 0.07$ & N.D. & N.D. & N.D. & N.D. & N.D. & N.D. \\
\hline & 6 & & $0.13 \pm 0.03$ & N.D. & N.D. & N.D. & N.D. & N.D. & N.D. \\
\hline & 7 & & $52.3 \pm 2.6$ & N.D. & N.D. & N.D. & N.D. & N.D. & N.D. \\
\hline & 8 & & $1.06 \pm 0.17$ & N.D. & N.D. & N.D. & N.D. & N.D. & N.D. \\
\hline & 9 & & $0.72 \pm 0.01$ & N.D. & N.D. & N.D. & N.D. & N.D. & N.D. \\
\hline
\end{tabular}




\begin{tabular}{|c|c|c|c|c|c|c|c|c|c|}
\hline & No. & & SAC & NEO & CYC & $\mathrm{ACE}$ & NHDC & SUC & ASP \\
\hline & 1 & sow & $1.60 \pm 0.17$ & N.D. & N.D. & N.D. & N.D. & N.D. & N.D. \\
\hline & 2 & hog & $81.4 \pm 1.1$ & N.D. & N.D. & N.D. & N.D. & N.D. & N.D. \\
\hline & 3 & hog & $11.4 \pm 0.9$ & N.D. & N.D. & N.D. & N.D. & N.D. & N.D. \\
\hline & 4 & piglet & $13.4 \pm 1.3$ & N.D. & N.D. & N.D. & N.D. & N.D. & N.D. \\
\hline & 5 & piglet & $6.25 \pm 0.74$ & N.D. & N.D. & N.D. & N.D. & 0.12 & N.D. \\
\hline & 6 & sow & $15.7 \pm 1.3$ & N.D. & N.D. & N.D. & N.D. & N.D. & N.D. \\
\hline & 7 & sow & $76.2 \pm 3.3$ & N.D. & N.D. & N.D. & N.D. & N.D. & N.D. \\
\hline & 8 & hog & $4.10 \pm 0.65$ & N.D. & N.D. & N.D. & N.D. & N.D. & N.D. \\
\hline & 9 & piglet & $10.5 \pm 1.3$ & N.D. & N.D. & N.D. & N.D. & N.D. & N.D. \\
\hline & 10 & sow & $0.15 \pm 0.01$ & N.D. & N.D. & N.D. & N.D. & N.D. & N.D. \\
\hline & 11 & hog & $0.30 \pm 0.02$ & N.D. & N.D. & N.D. & N.D. & N.D. & N.D. \\
\hline & 12 & piglet & $0.41 \pm 0.02$ & N.D. & N.D. & N.D. & N.D. & N.D. & N.D. \\
\hline & 13 & piglet & $263 \pm 12$ & N.D. & N.D. & N.D. & N.D. & 1.01 & N.D. \\
\hline & 14 & sow & $30.1 \pm 3.3$ & N.D. & N.D. & N.D. & N.D. & N.D. & N.D. \\
\hline & 15 & sow & $3.45 \pm 0.43$ & N.D. & N.D. & N.D. & N.D. & N.D. & N.D. \\
\hline Japan & 16 & hog & $0.51 \pm 0.01$ & N.D. & N.D. & N.D. & N.D. & N.D. & N.D. \\
\hline & 17 & hog & $0.13 \pm 0.01$ & N.D. & N.D. & N.D. & N.D. & N.D. & N.D. \\
\hline & 18 & piglet & $19.8 \pm 1.1$ & N.D. & N.D. & N.D. & N.D. & 0.19 & N.D. \\
\hline & 19 & piglet & $30.7 \pm 1.3$ & N.D. & N.D. & N.D. & N.D. & 0.21 & N.D. \\
\hline & 20 & piglet & $6.22 \pm 0.52$ & N.D. & N.D. & N.D. & N.D. & 0.26 & N.D. \\
\hline & 21 & hog & $0.14 \pm 0.01$ & N.D. & N.D. & N.D. & N.D. & 0.25 & N.D. \\
\hline & 22 & hog & $0.12 \pm 0.01$ & N.D. & N.D. & N.D. & N.D. & 0.71 & N.D. \\
\hline & 23 & sow & $0.14 \pm 0.01$ & N.D. & N.D. & N.D. & N.D. & N.D. & N.D. \\
\hline & 24 & sow & $0.43 \pm 0.02$ & N.D. & N.D. & N.D. & N.D. & N.D. & N.D. \\
\hline & 25 & piglet & $2.59 \pm 0.27$ & N.D. & N.D. & N.D. & N.D. & N.D. & N.D. \\
\hline & 26 & sow & $3.48 \pm 0.31$ & N.D. & N.D. & N.D. & N.D. & N.D. & N.D. \\
\hline & 27 & hog & $1.32 \pm 0.06$ & N.D. & N.D. & N.D. & N.D. & N.D. & N.D. \\
\hline & 28 & piglet & $2.34 \pm 0.03$ & N.D. & N.D. & N.D. & N.D. & N.D. & N.D. \\
\hline & 29 & hog & $1.19 \pm 0.02$ & N.D. & N.D. & N.D. & N.D. & N.D. & N.D. \\
\hline & 30 & sow & $0.85 \pm 0.01$ & N.D. & N.D. & N.D. & N.D. & N.D. & N.D. \\
\hline
\end{tabular}

Data are expressed as mean \pm SD. 